\title{
High-Resolution SEM with Coupled Transmission Mode and EDX for Quick Characterization of Micro- and Nanocapsules for self-Healing Anti-Corrosion Coatings
}

\author{
Vasile-Dan Hodoroaba $^{1}$, Dilek Akcakayiran ${ }^{2}$, Dmitri Grigoriev ${ }^{2}$ and Dmitry G. Shchukin ${ }^{2}$ \\ 1. BAM Federal Institute for Materials Research and Testing, Division 6.8 Surface Analysis and \\ Interfacial Chemistry, Berlin, Germany. \\ 2. Max-Planck Institute of Colloids and Interfaces, Department Interfaces, Potsdam, Germany.
}

More and more sub-micro and nano-materials shall be "quickly", but accurately characterized with respect to their morphology, shape, size or size distribution, but also to the chemical composition as well by means of an SEM/EDX (Scanning Electron Microscopy / X-Ray Energy Dispersive Spectrometry) system. This undertaking is becoming successful indeed if the transmission mode at low voltages such as those at a SEM, i. e. mostly up to $30 \mathrm{kV}$, and EDX, respectively is employed. The transmission mode at conventional SEM can be "activated" firstly by re-defining the specimen to be analyzed and preparing it as a thin specimen, which is quasi-transparent to electrons and by finding a way of collecting only the transmitted electrons for analysis. The first issue can be easily realized by using the conventional TEM grids (and the respective sample preparation); the second one becomes feasible either (i) by placing a socalled STEM semiconductor detector directly under the thin specimen or (ii) by using a special transmission set-up [1], which enables to the conventional Everhart-Thornley detector to collect only transmitted electrons by blocking the direct collection of secondary electrons and guiding only the transmitted electrons onto it.

The low-voltage transmission approach is not exactly new, however, the applications in the field of nano- (sub-micro) particles have been just intensifying and gains high attention due to its metrological, dimensional measurement capabilities [2,3]. Hence, "upper" observation with high-resolution "In-Lens" or "Through-the-Lens" detectors can be combined, i.e. complete each other, with high-resolution transmission microscopy by just pressing a key. Furthermore, if EDX is now "activated" for the analysis of the thin specimens, high-resolution EDX is enabled [3]. Of course, especially for nano-objects, the EDX signals will be very weak. However, the employment of the newly developed large-area SDDs (silicon drift detector) can improve significantly the signal-to-noise ratio and ensures valuable elemental information over the X-ray spectra.

One representative example of specimens on which the benefits of such a method combination can be excellently demonstrated is innovative micro- and nanocapsules fabricated specially as depots for healing agents to be embedded in self-healing anti-corrosion coatings [4,5]. To a large extent the morphology, shape, size, degree of feeling of the particles with the corrosion inhibitor, i. e. qualitative elemental composition, could be investigated readily by implementing/combining high-resolution SEM in the "upper" observation mode (with e.g. an In-lens detector) and the transmission mode (T-SEM), see Figs. 1 and 2, with high-resolution EDX, see Fig. 2. Fascinating individual details supplied by each particular method contribute significantly to clarify the posing of the analytical questions above (see the notes in the figure captions). 
References:

[1] U Golla, B Schindler et al., J. of Microscopy 173 (1994), p. 219.

[2] E Buhr, N Senftleben et al., Meas. Sci. Technol. 20 (2009), p. 413.

[3] V-D Hodoroaba, S Benemann et al., Microsc. Microanal. 18 (Suppl 2) (2012), p. 1750.

[4] D G Shchukin, D O Grigoriev et al., Soft Matter. 6 (2010), p. 720.

[5] A Latnikova, D O Grigoriev et al., J. Phys. Chem. C 116 (2012), p. 8181.
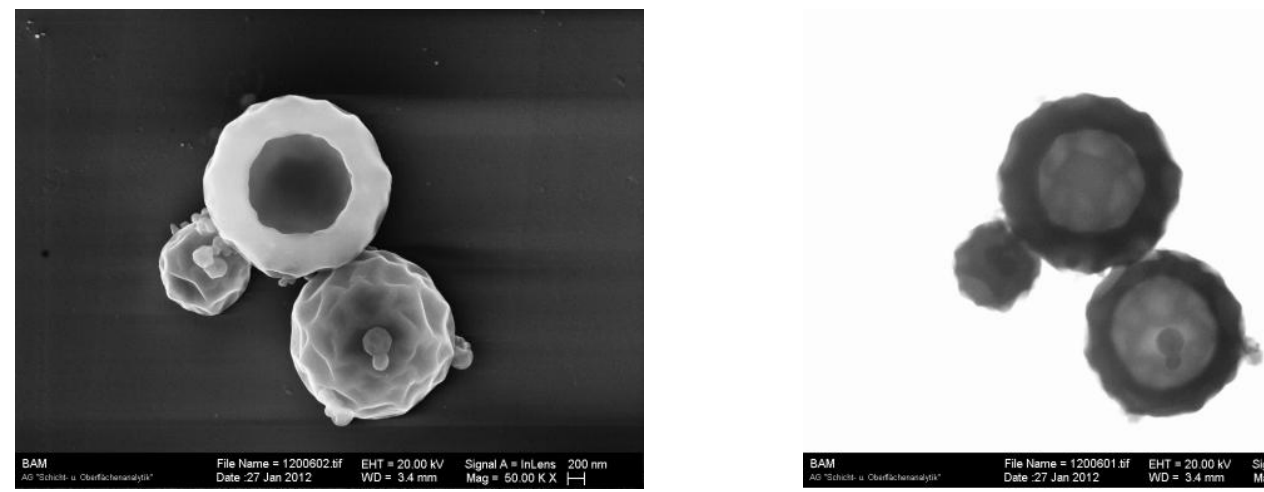

Figure 1. Example of complimentary "upper" observation of $\mathrm{SiO}_{2}$ (sub-)micro-capsules by the In-Lens detector (left) and transmission mode (right). Note the details on the surface in the left micrograph and the quite different occurrence of the big, bottom particle in the two microscopic modes.
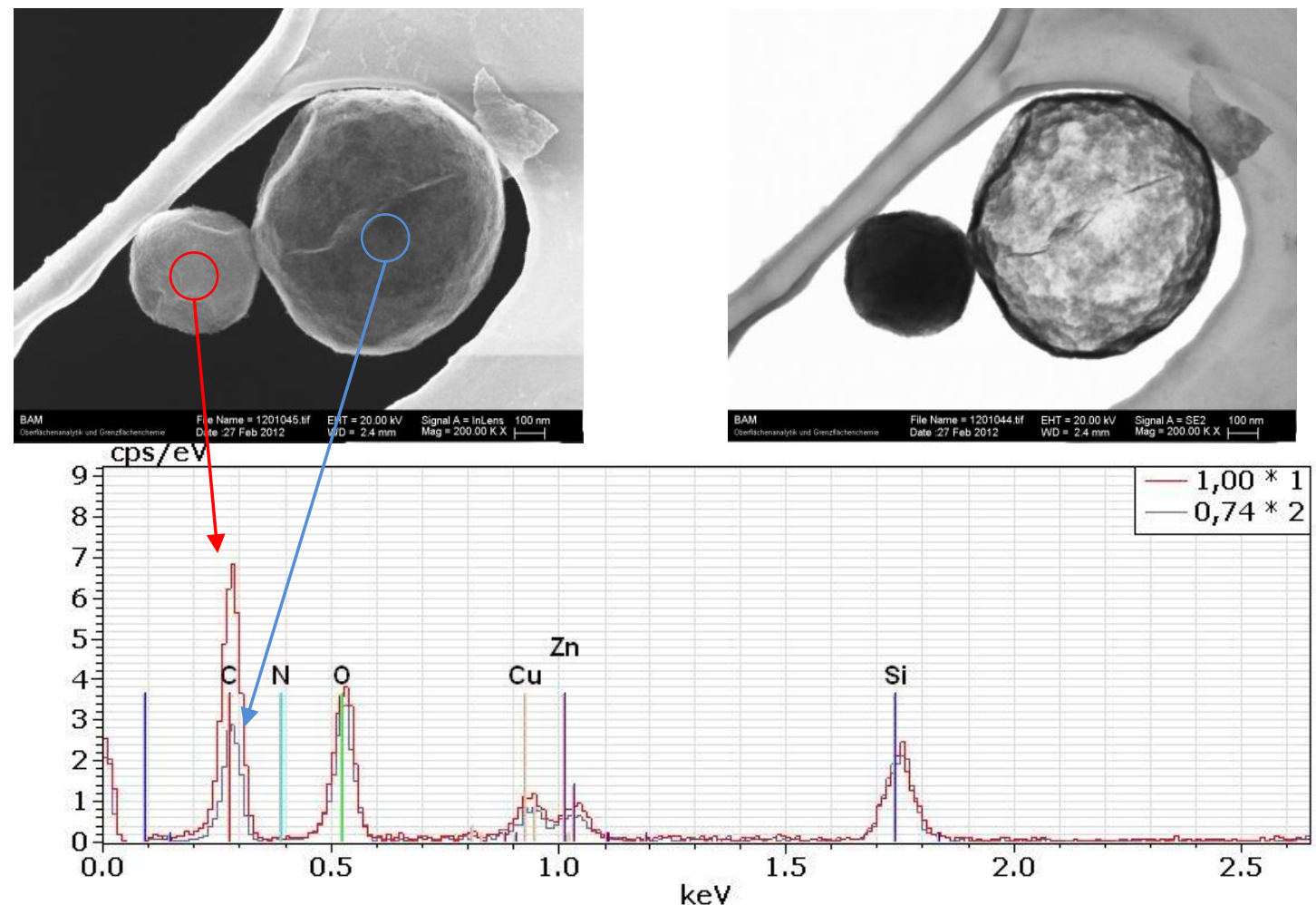

Figure 2. Upper (left) and in-transmission (right) $\mathrm{SEM}$ observation of $\mathrm{SiO}_{2}$ sub-micro-capsules. Note the clear elemental compositional difference between the two particles in the T-SEM mode (right). The presence of carbon as a main element "filling" the left particle is confirmed by EDX (bottom spectra). The right particle is constituted mainly by a $\mathrm{SiO}_{2}$ shell. $\mathrm{Cu}$ and $\mathrm{Zn}$ signals come from the TEM grid. 